\title{
Implicações nutricionais e tecnológicas da inclusão de antioxidantes em produtos
}

\section{lácteos}

\author{
Nutritional and technological implications of including antioxidants in dairy products \\ Implicaciones nutricionales y tecnológicas de la inclusión de antioxidantes en productos lácteos
}

Recebido: 21/09/2021 | Revisado: 29/09/2021 | Aceito: 30/09/2021 | Publicado: 03/10/2021

\author{
Melina Aparecida Plastina Cardoso \\ ORCID: https://orcid.org/0000-0002-0669-1469 \\ Universidade Estadual de Maringá, Brasil \\ E-mail: melina_cardoso@msn.com \\ Venicio Macedo Carvalho \\ ORCID: https://orcid.org/0000-0002-2875-7799 \\ Universidade Estadual de Maringá, Brasil \\ E-mail: venicio_@hotmail.com \\ Jessyca Caroline Rocha Ribas \\ ORCID: https://orcid.org/0000-0002-6046-2827 \\ Universidade Estadual de Maringá, Brasil \\ E-mail: jrocharibas@gmail.com \\ Bianka Rocha Saraiva \\ ORCID: https://orcid.org/0000-0002-6575-9857 \\ Universidade Estadual de Maringá, Brasil \\ E-mail: bianka_saraiva@hotmail.com \\ Fernando Antônio Anjo \\ ORCID: https://orcid.org/0000-0001-6886-0304 \\ Universidade Estadual de Maringá, Brasil \\ E-mail: fernandoaanjo@hotmail.com
}

\begin{abstract}
Resumo
Biologicamente, os antioxidantes possuem a função de inibirem ou ainda frearem processos de oxidação gerados naturalmente pelo sistema fisiológico das células. Tecnologicamente, são amplamente adicionados aos alimentos, cosméticos e fármacos, não somente com o intuito de retardar reações de oxidação e aumentar a vida útil, como também, para combater os radicais livres e proteger o sistema fisiológico do corpo humano. O presente trabalho teve como objetivo investigar, através de revisão de literatura, o uso de antioxidantes naturais que podem ser adicionados tecnologicamente a produtos lácteos, como fontes de auxílio, não somente ao sistema fisiológico do corpo, como também, às propriedades físico-químicas, reológicas e sensoriais dos produtos. A adição de antioxidantes em qualquer tipo de produto alimentício, cosmético ou fármaco, deve ser minuciosamente avaliada e testada, de acordo com as características desejáveis do produto final, respeitando sempre a lesgislação vigente. Vários alimentos de origem vegetal são utilizados como fontes de antioxidantes para o enriquecimento nutricional e tecnológico de produtos. Estes ingredientes funcionais podem ser obtidos e aplicados por diversas técnicas, dependendo do objetivo da aplicação. Contudo, ainda pouco se sabe sobre o efeito in vivo desdes alimentos quando consumidos, necessitando mais pesquisas que abordem a real absorção destes compostos pelo organismo.
\end{abstract}

Palavras-chave: Compostos bioativos; Enriquecimento de alimentos; Iogurte; Queijos; Radicais livres.

\begin{abstract}
Biologically, antioxidants have the function of inhibiting or even slowing down oxidation processes naturally generated by the physiological system of cells. Technologically, they are widely added to foods, cosmetics, and pharmaceuticals, not only with the aim of delaying oxidation reactions and increasing their shelf life, but also to fight free radicals and protect the physiological system of the human body. The present work aimed to investigate, through a literature review, the use of natural antioxidants that can be technologically added to dairy products, as sources of aid, not only to the physiological system of the body, but also to the physical and chemical properties, rheological and sensory characteristics of products. The addition of antioxidants in any type of food, cosmetic or drug product must be carefully evaluated and tested, according to the desirable characteristics of the final product, always respecting current legislation. Several plant foods are used as sources of antioxidants for nutritional and technological enrichment of products. These functional ingredients can be obtained and applied by different techniques, depending on the purpose of the application. However, little is known about the in vivo effect of these foods when consumed, requiring further research to address the actual absorption of these compounds by the body.
\end{abstract}

Keywords: Bioactive compounds; Cheeses; Food fortification; Free radicals; Yogurt. 


\section{Resumen}

Biológicamente, los antioxidantes tienen la función de inhibir o incluso ralentizar los procesos de oxidación generados naturalmente por el sistema fisiológico de las células. Tecnológicamente, se añaden ampliamente a alimentos, cosméticos y productos farmacéuticos, no solo con el objetivo de retrasar las reacciones de oxidación y aumentar su vida útil, sino también para combatir los radicales libres y proteger el sistema fisiológico del cuerpo humano. El presente trabajo tuvo como objetivo investigar, a través de una revisión de la literatura, el uso de antioxidantes naturales que se pueden agregar tecnológicamente a los productos lácteos, como fuentes de ayuda, no solo al sistema fisiológico del cuerpo, sino también a las propiedades físicas y químicas, reológicas y sensoriales de los productos. La adición de antioxidantes en cualquier tipo de producto alimenticio, cosmético o farmacéutico debe ser cuidadosamente evaluada y probada, de acuerdo con las características deseables del producto final, respetando siempre la legislación vigente. Varios alimentos vegetales se utilizan como fuentes de antioxidantes para el enriquecimiento nutricional y tecnológico de los productos. Estos ingredientes funcionales pueden obtenerse y aplicarse mediante diferentes técnicas, dependiendo del propósito de la aplicación. Sin embargo, se sabe poco sobre el efecto in vivo de estos alimentos cuando se consumen, lo que requiere más investigación para abordar la absorción real de estos compuestos por el cuerpo.

Palabras clave: Compuestos bioactivos; Fortificación de alimentos; Quesos; Radicales libres; Yogur.

\section{Introdução}

Radicais livres são moléculas altamente reativas, que endogenamente são liberadas pelo organismo de forma aleatória e podem provocar ou resultar em reações de óxido-redução, que muitas vezes são consideradas maléficas ao organismo humano (Phaniendra, Jestadi \& Periyasamy, 2015). Em suas formas exógenas, são encontrados na poluição, na radiação UV, na atmosfera, entre outros, podendo ser prejudiciais ao corpo. Como forma de resposta a estes radicais livres, o sistema natural libera, de forma aleatória e seletiva, antioxidantes endógenos capazes de proteger o organismo de tais reações, reduzindo ou ainda inibindo a ação de substratos oxidativos (Nimse \& Pal, 2015). Embora o sistema natural de antioxidantes endógenos presentes no corpo esteja preparado para o estresse oxidativo que ele mesmo produz, estes não são suficientemente capazes de combatê-lo (Halliwell, 2011).

Sob essa perspectiva, antioxidantes que possam ser incluídos em alimentos vêm sendo estudados como uma fonte alternativa de apoio aos antioxidantes endógenos (Lorenzo, Manuel, Trindade, Ahn \& Barba, 2019). Em alimentos, os antioxidantes possuem diversas funções, como por exemplo: são capazes de aumentar a vida útil, melhorar a cor e o sabor, preservar a qualidade de macronutrientes, suprir reações de oxidação de componentes insaturados, participar ou cessar a autoxidação lipídica por meio de diversos mecanismos (Ahmed, Saaed \& Marhoon, 2018; Tian, Decker \& Goddard, 2013).

Diante deste contexto, o presente trabalho teve como objetivo investigar, através de uma revisão de literatura, o uso de antioxidantes naturais que podem ser adicionados tecnologicamente a produtos lácteos, como fontes de auxílio, não somente ao sistema endógeno do corpo humano, como também, às propriedades físico-químicas, reológicas e sensoriais dos produtos.

\section{Metodologia}

Para realizar a busca das produções científicas foram utilizados os seguintes descritores: free radicals, antioxidants, oxidation, milk products, cheese, yogurt, publicados no período compreendido entre 2011 e 2021. Definiu-se, como critérios de inclusão, que tais descritores estivessem presentes ou no resumo ou no título do trabalho.

Foram considerados como instrumentos de apoio à pesquisa: livros, artigos, revistas, trabalhos acadêmicos, periódicos ou relatórios que estivessem inseridos no "Google acadêmico" e que apresentassem data de publicação dentro do período prédeterminado. Outros materiais que serviram de apoio à pesquisa também foram considerados, como por exemplo: periódicos contidos na base de dados da Scielo, notícias, relatórios e guias de cunho governamental, como também diários oficiais e legislações pertinentes, publicados em qualquer ano, porém, considerados pertinentes e relevantes ao estudo. Excluiu-se as publicações em teses, dissertações e aquelas que não atendessem aos objetivos propostos ou que não estavam alinhadas aos critérios de inclusão. 


\section{Resultados e Discussão}

\subsection{O que são radicais livres e como atuam?}

Radicais livres são moléculas produzidas e liberadas pelo organismo humano consideradas extremamente instáveis e altamente reativas, que se combinam com outras moléculas do corpo, causando interações. A potencialidade de sua reatividade está diretamente ligada a instabilidade da molécula, que apresenta um desemparelhamento de elétrons na última camada (número ímpar de elétrons em sua camada eletrônica) (Halliwell \& Gutteridge, 2015).

Os radicais livres são formados a partir de um cenário de reações de óxido-redução, ou seja, são capazes de ceder um elétron solitário (desemparelhado) e então, sofrem oxidação; ou ainda podem receber um elétron e sofrer redução (Nimse \& Pal, 2015). Como exemplos de radicais livres encontrados na natureza temos: o radical hidroxila, radical proxil, ácido nítrico, ânion peróxido, radical alcoxil, entre outros (Phaniendra, Jestadi \& Periyasamy, 2015).

Os radicais livres mais conhecidos são o $\mathrm{OH}$ e o $\mathrm{O}_{2}^{2}$, sendo formados no interior das mitocôndrias a partir do processo normal ou acelerado da redução de oxigênio molecular. Estes processos são considerados vitais por gerar energia através da geração de ATP (adenosina trifosfato) em organismos aeróbios (Sharma, Gupta \& Sharma, 2018). Os radicais livres possuem um importante papel para o sistema imune ao apresentar ações antimicrobianas (Poprac et al., 2017).

Existem as fontes exógenas de geração ou indução dos radicais livres, como a radiação UV, poluição, dietas ingeridas de forma irregular e extremamente calórica, pesticidas entre outros que podem afetar o organismo (Neha, Haider, Pathak \& Yar, 2019). O excesso de radicais livres no corpo humano podem acarretar em danos irreversíveis como o aparecimento e desenvolvimento de doenças (Parkinson, Alzheimer, câncer) ou ainda, envelhecimento precoce (Nimse \& Pal, 2015).

\subsection{Antioxidantes: principais características, tipos e vias de atuação}

Há uma infinidade de antioxidantes estudados no intuito de prevenirem algumas doenças que acreditam ser causadas por estresse oxidativo gerado através de espécies reativas de oxigênio. As doenças mais citadas na literatura são: doenças cardiovasculares, câncer, diabetes, neurodegenerativas (como por exemplo, Parkinson, o mal de Alzheimer e a doença de Huntington), envelhecimento, entre outras (Szymanska, Pospíšil \& Kruk, 2018).

Os antioxidantes advindos da dieta são primordiais para a manutenção da saúde dos seres humanos (Crowe-White et al., 2017). Os antioxidantes quando ingeridos de maneira adequada podem limitar a oxidação sistêmica e ainda, manter a proporção correta de glutationa reduzida para oxidada, sendo responsáveis por prevenir doenças cardiovasculares (Varadharaj et al., 2017). Vitaminas como a C, D e E tem efeito protetor à doença de Parkinson. Com destaque a vitamina D que comprovadamente age na atenuação da deterioração da doença de Parkinson e redução da ocorrência de fraturas nos pacientes suplementados (Zhao et al., 2019).

Os flavonoides protegem o organismo dos danos causados ao DNA pela ação de radicais hidroxila através de mecanismos de quelação. A adição de especiarias e ervas na alimentação como a curcumina, rica em flavonoides, podem contribuir na prevenção de doenças cardiovasculares e câncer (Griffiths et al. 2016; Halliwell 2012).

Antioxidantes como ácido alfa lipóico apresentam-se eficientes contra diabetes mellitus (Golbidi, Badran \& Laher, 2011; Rochette, Ghibu, Muresan \& Vergely, 2015). Outros tipos de antioxidantes naturais também apresentam capacidade de melhorar o controle da glicemia e prevenção de diabetes mellitus como a inulina (Gargari, Dehghan, Aliasgharzadeh \& JafarAbadi, 2013), o extrato da uva espim (Berberis vulgaris) (Shidfar et al., 2012), chá de camomila (Zemestani, Rafraf \& Asghari-Jafarabadi, 2016) e o extrato das ervas Ginkgo biloba e Camellia sinensis (Lasaite, Spadiene, Savickiene, Skesters \& Silova, 2014). Sendo assim, os antioxidantes são considerados benéficos, não somente no auxílio a diabetes, como também para o trato gastrointestinal, perfil lipídico, função renal, dentre outros (Thakur, Kumar \& Kumar, 2018). 
Porém, é baixa a ingestão de compostos antioxidantes pela população, fato comprovado em pesquisa com 33 mil entrevistados, principalmente em relação as vitaminas e minerais quando questionado a pessoas abaixo do peso ideal (Tureck, Locateli, Côrrea \& Koehnlein, 2017). Observou-se, ainda, que os entrevistados possuem a consciência dos nutrientes antioxidantes presentes nos alimentos ingeridos (vegetais, frutas, legumes) e associam este fato a qualidade da dieta.

Os grupos de antioxidantes mais estudados são: ácidos fenólicos, os flavonoides, carotenoides, compostos organossulfurados, enzimas, vitaminas e os minerais. Podem estar presentes (de forma sintética ou naturais) tanto em plantas, quanto em fármacos e/ou em alimentos (Vassalle et al., 2017). São tecnologicamente utilizados na forma de pó, extratos lipossolúveis ou hidrossolúveis, encapsulados, como ingredientes fortificantes ou biofortificantes, em coberturas comestíveis ou adicionados propositalmente em embalagens ativas entre outros (Zehiroglu \& Sarikaya, 2019).

Os antioxidantes podem ser lipossolúveis (tocoferol, carotenoides, ubiquinol, bilirrubina, etc.) ou hidrossolúveis (ácido ascórbico, ácido úrico, glutationa, cisteína, etc.), atuando na prevenção ou correção de sistemas, obtidos de fonte natural ou sintética (Nimse \& Pal, 2015; Surai, 2020). As estruturas químicas desses compostos podem ser vistas na Figura 1.

Figura 1 - Estruturas químicas de antioxidantes lipossolúveis e hidrossolúveis.
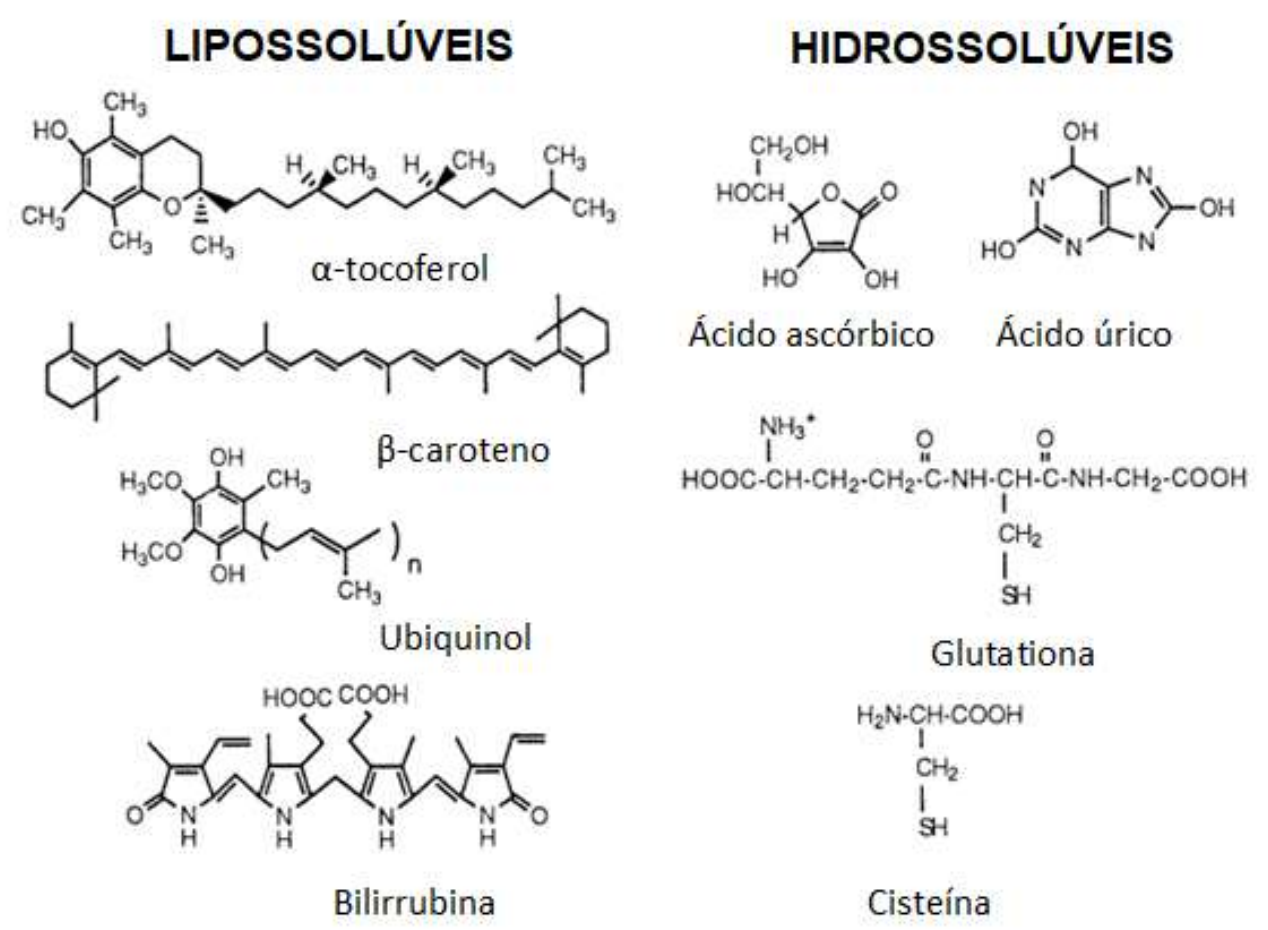

Fonte: Adaptado de Krinsky (1992).

Considerando o seu modo de ação, os antioxidantes são classificados como primários ou secundários. Os primários interrompem a cadeia de reação do radical livre, doando elétrons ou hidrogênios a estes radicais, (gerando produtos termodinamicamente estáveis) e/ou ligando-se com os radicais livres, formando complexos capazes de reagir com outros radicais livres. Já os antioxidantes secundários atuam retardando as etapas iniciais de autoxidação, através da complexação de metais, sequestro de oxigênio, absorção de radiações ultravioletas, desativação de oxigênios singletes ou ainda, decompondo hidroperóxidos (Pisoschi \& Pop, 2015).

Os compostos antioxidantes ao combater os radicais livres, protegem o corpo contra doenças degenerativas, diminuindo sua incidência e prevenindo contra atividades altamente reativas de oxigênios (Amiri \& Amiri, 2017). 
Biologicamente, a função básica dos antioxidantes baseia-se em atuar como protetores endógenos capazes de reduzir ou inibir a atuação de determinado substrato oxidativo, mesmo quando presente em concentrações menores que a do substrato. Tais atuações podem se concentrar em redução do estresse oxidativo, mutações de DNA, transformações bioquímicas entre outras (Halliwell \& Gutteridge, 2015).

O equilíbrio entre a produção de radicais livres e a produção e acionamento das defesas antioxidantes é fundamental para a manutenção da saúde. Os antioxidantes atuam através de defesas enzimáticas (quando envolvem enzimas como superóxido dismutase - SOD, glutadiona peroxidade - $\mathrm{GPx}^{23}$, catalase - CAT, que agem sob o acúmulo de radicais peróxidos e superóxidos de hidrogênio) ou não enzimáticas, incluindo produtos sintéticos (BHA, BHT, PG e TBHQ) ou naturais (Vitaminas A, C e E, polifenóis, flavonoides, carotenoides, extratos de plantas, etc.) (Halliwell, 2011; Alkadi, 2020).

As enzimas que atuam como antioxidantes são capazes de bloquear a reação da oxidação por interagir com as moléculas reativas de oxigênio. A atividade não enzimática interagem com espécies radicalares, sendo consumidas durante a reação (Carocho, Morales \& Ferreira, 2018).

\subsection{Antioxidantes em produtos alimentícios}

A autoxidação é um processo comumente observado em vitaminas C e E que se decompõem em ácido desidroascórbico e radical tocoferoxil, respectivamente. Nos óleos e gorduras há a reação da peroxidação lipídica (mediada de forma espontânea pela enzima lipoxidase) dos ácidos graxos insaturados, colesterol e fosfolipídios (Carocho \& Ferreira, 2013; Ahmed et al., 2018). A oxidação pode alterar as propriedades organolépticas dos alimentos, conferindo o sabor de ranço e reduzindo sua aceitação (Lorenzo et al., 2019). Os efeitos da oxidação resultam na diminuição da cor (atributo importante em relação à apresentação do produto), em problemas na textura, na diminuição do valor nutricional e na formação de compostos indesejáveis e tóxicos, como por exemplo, cetonas, peróxidos e etc. (Tian, Decker \& Goddard, 2013; Hu \& Jacobsen, 2016).

Os antioxidantes podem estar presentes nos alimentos de forma natural ou ser adicionados. Neste último, os compostos devem ser atóxicos e não conferir características indesejáveis aos alimentos. Suas aplicações, quantidades utilizadas e tecnologias de adição diferem conforme o alimento, variam de acordo com as características desejáveis ao produto final e conforme legislações específicas (Carocho et al. , 2018).

Diversas técnicas de adição de diferentes antioxidantes podem ser encontradas na literatura. Tais antioxidantes podem ser acrescentados em produtos cárneos (carnes vermelhas, carnes brancas, pescados, hambúrgueres, etc.), produtos vegetais (in natura ou processados, como sucos e polpas), ovos, produtos à base de cereais (pães, bolos, tortas, etc.) e ainda, produtos lácteos (Vandamme \& Revuelta, 2016; Laganà et al., 2017; Zehiroglu \& Sarikaya, 2019; Granato et al., 2020).

\subsection{Principais antioxidantes utilizados em produtos lácteos}

Produtos lácteos são aqueles que possuem o leite como ingrediente principal na sua composição na forma de fluido pasteurizado, esterilizado, desnatado ou semidesnatado (Khan et al., 2019). Dentro desta classificação, existe uma infinidade de produtos que podem ser listados contendo uma legislação específica que os rege (MAPA, 2020).

O consumo adequado de leite e derivados contribuem com micro e macronutrientes essenciais para a dieta humana, sem restrição etária, exceto quando há condições médicas específicas como intolerância à lactose ou alergia à proteína do leite (Marangoni et al., 2019).Além disso, várias pesquisas relacionam o consumo do leite com a prevenção de diversas enfermidades (Aune et al., 2012; Davoodi, Esmaeili \& Mortazavian, 2013).

Produtos lácteos geralmente exibem maior atratividade por parte dos consumidores, pois podem ser elaborados com diferentes sabores e texturas. Iogurtes, kefir, coalhada e outros produtos produzidos através da fermentação do leite exibem 
maior digestibilidade quando comparado ao leite fresco e maior shelf-life pois o processo de fermentação do leite resulta em produção de ácido lático (Finco, Garmus, Bezerra \& Córdova, 2011).

A adição de ingredientes com capacidade antioxidante em produtos lácteos é bastante estudada (Fidelis et al., 2020; Muniandy, Shori \& Baba, 2016; Ribas et al., 2019). Estes, tem capacidade de melhorar as condições nutricionais dos produtos. A Tabela 1 apresenta alguns trabalhos realizados com o intuito de adicionar antioxidantes naturais em produtos lácteos.

Tabela 1. Antioxidantes em produtos lácteos.

\begin{tabular}{|c|c|c|}
\hline Produto lácteo & Conclusão & Referências \\
\hline Iogurte & $\begin{array}{l}\text { A adição de chá verde, branco ou preto (Camellia sinensis) em } \\
\text { iogurtes aumentou o potencial antioxidante por meio de } \\
\text { eliminação do radical DPPH, poder redutor férrico e quelante de } \\
\text { íons ferrosos. }\end{array}$ & Muniandy et al.,2016 \\
\hline Queijo petit suisse & $\begin{array}{l}\text { A inclusão de extrato de jabuticaba (Plinia cauliflora) em queijos } \\
\text { do tipo "petit suisse" melhorou suas propriedades antioxidantes } \\
\text { sem afetar a composição físico-química do produto, entretanto os } \\
\text { queijos apresentaram comportamento pseudoplástico. }\end{array}$ & Pereira et al., 2016 \\
\hline Iogurte & $\begin{array}{l}\text { A utilização de bagaço de uva (Vitis labrusca L.) desidratada } \\
\text { aumenta a atividade antirradical do iogurte sem modificar o } \\
\text { crescimento de bactérias ácido láticas. }\end{array}$ & Demirkol \& Tarakci, 2018 \\
\hline Queijo tipo macio & $\begin{array}{l}\text { Ao adicionar diretamente no leite, extratos de casca de } \\
\text { amendoim, casca de batata e farelo de arroz geraram um queijo } \\
\text { rico em antioxidantes com baixa quantidade de peróxidos. }\end{array}$ & El-Galeel, Ali \& El-Zahar, 2018 \\
\hline Queijo Gouda & $\begin{array}{l}\text { A substituição parcial da gordura do queijo Gouda por gordura } \\
\text { do caroço de manga aumentou a atividade sequestrante de } \\
\text { radicais livres, melhorou a estabilidade oxidativa e características } \\
\text { sensoriais. }\end{array}$ & $\begin{array}{l}\text { Khan, Nadeem, Imran, Ajmal } \\
\text { \& Ali, } 2018\end{array}$ \\
\hline Pasta de queijo & $\begin{array}{l}\text { A inclusão de bagaço de uva branca e roxa em pasta de queijo } \\
\text { resultou em melhores resultados nutricionais e sensoriais além de } \\
\text { maior capacidade antioxidante. }\end{array}$ & Lucera et al., 2018 \\
\hline $\begin{array}{l}\text { Queijo do tipo } \\
\text { "feta" }\end{array}$ & $\begin{array}{l}\text { Ao adicionar diretamente no leite, extratos de casca de romã } \\
\text { demonstraram alta atividade antioxidante nos queijos com } \\
\text { diminuição da quantidade de peróxidos e redução significativa de } \\
\text { bactérias totais, coliformes e fungos }\end{array}$ & $\begin{array}{l}\text { Khalil, El-Wahed, Shalaby \& } \\
\text { Gaballa, } 2019\end{array}$ \\
\hline Iogurte & $\begin{array}{l}\text { O extrato de semente de chia melhorou as propriedades bioativas } \\
\text { do iogurte, acelerou significativamente a taxa de fermentação e o } \\
\text { crescimento de bactérias ácido láticas. }\end{array}$ & Kwon, Bae, Seo \& Han, 2019 \\
\hline Queijo frescal & $\begin{array}{l}\text { A inclusão de manjericão (Ocimum basilicum Lamiaceae) em } \\
\text { queijo frescal elaborado com leite de búfala resultou em maior } \\
\text { atividade antioxidante e menor pH afetando a dureza e } \\
\text { mastigabilidade do produto. }\end{array}$ & Ribas et al., 2019 \\
\hline Queijo frescal & $\begin{array}{l}\text { A inclusão de erva mate (Ilex paraguariensis A. St.-Hil.) em } \\
\text { queijos frescais resultou em maior atividade antioxidante, com } \\
\text { efeitos na cor, textura e estrutura dos queijos. }\end{array}$ & $\begin{array}{l}\text { Saraiva, Vital, Anjo, Ribas \& } \\
\text { Matumoto-Pintro, } 2019\end{array}$ \\
\hline Iogurte & $\begin{array}{l}\text { A adição de extrato de semente de camu-camu (myrciaria dubia) } \\
\text { aumentou a atividade antioxidante do iogurte e teve alto índice de } \\
\text { aceitação pelos provadores. }\end{array}$ & Fidelis et al., 2020 \\
\hline Queijos frescal & $\begin{array}{l}\text { O acréscimo de extratos de erva-mate e chá branco em queijos } \\
\text { embalados com filmes comestíveis melhorou suas propriedades } \\
\text { antioxidantes e a qualidade organoléptica dos queijos, quando } \\
\text { comparados ao controle. }\end{array}$ & $\begin{array}{l}\text { Pluta-Kubica, Jamróz, } \\
\text { Kawecka, Juszczak \& Krysciak, } \\
2020\end{array}$ \\
\hline
\end{tabular}

Fonte: Autores.

Conforme apresentado na tabela, vários pesquisadores têm buscado adicionar antioxidantes naturais em produtos lácteos visando aumentar sua vida de prateleira, melhorar suas características nutricionais ou ainda estudar a relação da adição destes antioxidantes nas características reológicas, microbiológicas e sensoriais do derivado lácteo. Em produtos lácteos do 
tipo queijo, a adição de antioxidantes têm se mostrado benéfica e eficiente, tanto na adição direta ao leite utilizado na formulação, como também na adição direta ao produto final e ainda, na adição de coberturas e/ou embalagens ativas. Tais adições apresentam-se eficientes, pois são capazes de diminuir a oxidação lipídica em queijos, aumentando sua vida de prateleira e, ainda, preservar características sensoriais.

No caso de iogurtes, além de aumentar a atividade antioxidante do produto final, a inclusão de compostos bioativos pode auxiliar no desenvolvimento dos microrganismos fermentadores, acelerando o processo de fermentação. Também há benefícios tecnofuncionais devido as interações polifenois-proteínas que ajudam na estrtuturação do iogurte, dificultando a sinerese. Esta melhor estruturação também contribui para a melhoria das propriedades reológicas e de textura do produto.

Cabe salientar que estes compostos podem alterar as características sensoriais do produto final e interferir na aceitabilidade do consumidor, desta forma, a análise sensorial é essencial para a formulação de novos produtos.

\section{Conclusão}

Extratos ricos em compostos bioativos obtidos de partes de frutas, ervas, especiarias e diversos alimentos, vêm sendo utilizados como antioxidantes para o enriquecimento nutricional e tecnológico de diversos produtos. A aplicação destes extratos pode ser realizada de diversas formas, possibilitando, não apenas o desenvolvimento de novos produtos, como também o de novos ingredientes, os quais podem apresentar-se em diferentes formas físicas e, portanto, originando diferentes formas de aplicações. Contudo, a maioria dos trabalhos abordam os efeitos da adição dos ingredientes funcionais (antioxidantes) in vitro, sendo necessário a busca pelo entendimento da ação destes antioxidantes quando ingeridos na forma de alimentos processados, ou seja, avaliar a ação antioxidante destes alimentos ricos em compostos bioativos in vivo.

\section{Referências}

Ahmed, L. M., Shaymaa I. S., \& Ashraff A. M. 2018. "Effect of Oxidation Agents on Photo-Decolorization of Vitamin B12 in the Presence of ZnO/UV-A System.” Indonesian Journal of Chemistry 18(2):272. doi: 10.22146/ijc.33470.

Alkadi, H. 2020. “A Review on Free Radicals and Antioxidants.” Infectious Disorders - Drug Targets 20(1):16-26. doi: $10.2174 / 1871526518666180628124323$.

Amiri, A., \& Armin A. 2017. "Antioxidants and Disease Prevention; an Obscure Association with Great Significance.” Annals of Research in Antioxidants 2(1).

Aune, D., R. Lau, D. S. M. Chan, R. Vieira, D. C. Greenwood, E. Kampman, \& T. Norat. 2012. "Dairy Products and Colorectal Cancer Risk: A Systematic Review and Meta-Analysis of Cohort Studies." Annals of Oncology 23(1):37-45. doi: 10.1093/annonc/mdr269.

Carocho, M., \& Isabel C. F. R. Ferreira. 2013. "A Review on Antioxidants, Prooxidants and Related Controversy: Natural and Synthetic Compounds, Screening and Analysis Methodologies and Future Perspectives.” Food and Chemical Toxicology 51:15-25. doi: 10.1016/j.fct.2012.09.021.

Carocho, M., Patricia M., \& Isabel C. F. R. Ferreira. 2018. “Antioxidants: Reviewing the Chemistry, Food Applications, Legislation and Role as Preservatives." Trends in Food Science \& Technology 71:107-20. doi: 10.1016/j.tifs.2017.11.008.

Crowe-White, K., J. Scott P., Kim S. Stote, Melissa Gutschall, Sue Benson-Davies, Elizabeth Droke, Carol E. O’Neil, Taylor Wolfram, \& Paula Ziegler. 2017. "Metabolic Impact of 100\% Fruit Juice Consumption on Antioxidant/Oxidant Status and Lipid Profiles of Adults: An Evidence-Based Review." Critical Reviews in Food Science and Nutrition 57(1):152-62. doi: 10.1080/10408398.2015.1102861.

Davoodi, H., S. Esmaeili, \& A. M. Mortazavian. 2013. "Effects of Milk and Milk Products Consumption on Cancer: A Review." Comprehensive Reviews in Food Science and Food Safety 12(3):249-64. doi: 10.1111/1541-4337.12011.

Demirkol, Melike \& Tarakci, Zekai. (2018). Effect of grape ( Vitis labrusca L.) pomace dried by different methods on physicochemical, microbiological and bioactive properties of yoghurt. LWT. 97. 10.1016/j.1wt.2018.07.058.

El-Galeel, Abd, A. A. Ali, \& Kahled M. El-Zahar. 2018. "Production of White Soft Cheese Fortified with Natural Antioxidants as a Functional Dairy Food." Zagazig Journal of Agricultural Research 45(2):637-56.

Fidelis, Marina, Stephanie Maiara Oliveira, Jânio Sousa Santos, Graziela Bragueto Escher, Ramon Silva Rocha, Adriano Gomes Cruz, Mariana Araújo Vieira Carmo, Luciana Azevedo, Tai Kaneshima, Won Young Oh, Fereidoon Shahidi, \& Daniel Granato. 2020. "From Byproduct to a Functional Ingredient: CamuCamu (Myrciaria Dubia) Seed Extract as an Antioxidant Agent in a Yogurt Model.” Journal of Dairy Science 103(2):1131-40. doi: 10.3168/jds.2019-17173.

Finco, Ana Maria Oliveira, Tábata Tayara Garmus, José Raniere Mazile Vidal Bezerra, \& Katielle Rosalva Voncik Córdova. 2011. Elaboração de Iogurte Com Adição de Farinha de Gergelim. Ambiência 7(2):217-27. 
Gargari, Bahram Pourghassem, Parvin Dehghan, Akbar Aliasgharzadeh, \& Mohammad Asghari Jafar-abadi. 2013. "Effects of High Performance Inulin Supplementation on Glycemic Control and Antioxidant Status in Women with Type 2 Diabetes." Diabetes \& Metabolism Journal 37(2):140. doi: 10.4093/dmj.2013.37.2.140.

Golbidi, Saeid, Mohammad Badran, \& Ismail Laher. 2011. "Diabetes and Alpha Lipoic Acid.” Frontiers in Pharmacology 2. doi: 10.3389/fphar.2011.00069.

Granato, Daniel, Francisco J. Barba, Danijela Bursać Kovačević, José M. Lorenzo, Adriano G. Cruz, \& Predrag Putnik. 2020. "Functional Foods: Product Development, Technological Trends, Efficacy Testing, and Safety." Annual Review of Food Science and Technology 11(1):93-118. doi: 10.1146/annurevfood-032519-051708.

Griffiths, Keith, Bharat B. Aggarwal, Ram B. Singh, Harpal S. Buttar, Douglas Wilson, \& Fabien Meester. 2016. "Food Antioxidants and Their AntiInflammatory Properties: A Potential Role in Cardiovascular Diseases and Cancer Prevention." Diseases 4(3):28.

Halliwell, B., \& J. M. C. Gutteridge. 2015. Free Radicals in Biology and Medicine. Oxford University Press.

Halliwell, Barry. 2011. "Free Radicals and Antioxidants - Quo Vadis?” Trends in Pharmacological Sciences 32(3):125-30. doi: 10.1016/j.tips.2010.12.002.

Halliwell, Barry. 2012. "Free Radicals and Antioxidants: Updating a Personal View." Nutrition Reviews 70(5):257-65. doi: 10.1111/j.17534887.2012.00476.x.

Hu, M., \& C. Jacobsen. 2016. Oxidative Stability and Shelf Life of Foods Containing Oils and Fats. Elsevier Science.

Khalil, Merehan, Abd El-Wahed, Hanan S. Shalaby, \& A. S. Gaballa. 2019. "Production of Feta like Cheese Fortified with Pomegranate and Lemon Peels Extract as Natural Antioxidants." Zagazig Journal of Agricultural Research 46(3):710-20.

Khan IT, Nadeem M, Imran M, Ajmal M, Ali S. 2018. Antioxidant activity, fatty acids characterization and oxidative stability of Gouda cheese fortified with mango (Mangifera indica L.) kernel fat. J Food Sci Technol. 55(3):992-1002. doi: 10.1007/s13197-017-3012-y.

Khan, Imran Taj, Muhammad Nadeem, Muhammad Imran, Rahman Ullah, Muhammad Ajmal, and Muhammad Hayat Jaspal. 2019. "Antioxidant Properties of Milk and Dairy Products: A Comprehensive Review of the Current Knowledge." Lipids in Health and Disease 18(1):41. doi: 10.1186/s12944-019-0969-8.

Krinsky, Norman I. 1992. "Mechanism of Action of Biological Antioxidants." Experimental Biology and Medicine 200(2):248-54.

Kwon HC, Bae H, Seo HG, \& Han SG. Short communication: Chia seed extract enhances physiochemical and antioxidant properties of yogurt. J Dairy Sci. 2019 Jun;102(6):4870-4876. doi: 10.3168/jds.2018-16129. Epub 2019 Apr 17. PMID: 31005316.

Laganà, Pasqualina, Emanuela Avventuroso, Giovanni Romano, Maria Eufemia Gioffré, Paolo Patanè, Salvatore Parisi, Umberto Moscato, \&Santi Delia. 2017. "Classification and Technological Purposes of Food Additives: The European Point of View." Pp. 1-21 in SpringerBriefs in Molecular Science.

Lasaite, Lina, Asta Spadiene, Nijole Savickiene, Andrejs Skesters, and Alise Silova. 2014. "The Effect of Ginkgo Biloba and Camellia Sinensis Extracts on Psychological State and Glycemic Control in Patients with Type 2 Diabetes Mellitus." Natural Product Communications 9(9):1934578X1400900. doi: $10.1177 / 1934578 X 1400900931$

Lorenzo, Jose Manuel, Marco Antonio Trindade, Dong Uk Ahn, \& Francisco J. Barba. 2019. "Natural Antioxidants to Reduce the Oxidation Process of Meat and Meat Products." Food Research International 115:377-78. doi: 10.1016/j.foodres.2018.11.015.

Lucera, Annalisa, Cristina Costa, Valeria Marinelli, Maria Antonietta Saccotelli, Matteo Alessandro Del Nobile, \& Amalia Conte. 2018. "Fruit and Vegetable By-Products to Fortify Spreadable Cheese." Antioxidants 7(5). doi: 10.3390/antiox7050061.

MAPA. 2020. "Legislação Produtos Lácteos.” Ministério Da Agricultura, Pecuária e Abastecimento, 1-2.

Marangoni, Franca, Luisa Pellegrino, Elvira Verduci, Andrea Ghiselli, Roberto Bernabei, Riccardo Calvani, Irene Cetin, Michelangelo Giampietro, Francesco Perticone, Luca Piretta, Rosalba Giacco, Carlo La Vecchia, Maria Luisa Brandi, Donatella Ballardini, Giuseppe Banderali, Stefano Bellentani, Giuseppe Canzone, Claudio Cricelli, Pompilio Faggiano, Nicola Ferrara, Evelina Flachi, Stefano Gonnelli, Claudio Macca, Paolo Magni, Giuseppe Marelli, Walter Marrocco, Vito Leonardo Miniello, Carlo Origo, Filomena Pietrantonio, Paolo Silvestri, Roberto Stella, Pasquale Strazzullo, Ersilia Troiano, \& Andrea Poli. 2019. "Cow's Milk Consumption and Health: A Health Professional's Guide." Journal of the American College of Nutrition 38(3):197-208. doi: $10.1080 / 07315724.2018 .1491016$.

Muniandy, Premalatha, Amal Bakr Shori, \& Ahmad Salihin Baba. 2016. "Influence of Green, White and Black Tea Addition on the Antioxidant Activity of Probiotic Yogurt during Refrigerated Storage." Food Packaging and Shelf Life 8:1-8. doi: 10.1016/j.fps1.2016.02.002.

Neha, Kumari, Md Rafi Haider, Ankita Pathak, \& M. Shahar Yar. 2019. "Medicinal Prospects of Antioxidants: A Review." European Journal of Medicinal Chemistry 178:687-704. doi: 10.1016/j.ejmech.2019.06.010.

Nimse, Satish Balasaheb, \& Dilipkumar Pal. 2015. "Free Radicals, Natural Antioxidants, and Their Reaction Mechanisms." RSC Advances 5(35):27986-6.

Pereira, E. P. R., R. N. Cavalcanti, E. A. Esmerino, R. Silva, L. R. M. Guerreiro, R. L. Cunha, H. M. A. Bolini, M. A. Meireles, J. A. F. Faria, \& A. G. Cruz. 2016. "Effect of Incorporation of Antioxidants on the Chemical, Rheological, and Sensory Properties of Probiotic Petit Suisse Cheese." Journal of Dairy Science 99(3):1762-72. doi: 10.3168/jds.2015-9701.

Phaniendra, Alugoju, Dinesh Babu Jestadi, \& Latha Periyasamy. 2015. "Free Radicals: Properties, Sources, Targets, and Their Implication in Various Diseases." Indian Journal of Clinical Biochemistry 30(1):11-26. doi: 10.1007/s12291-014-0446-0.

Pisoschi, Aurelia Magdalena, \& Aneta Pop. 2015. "The Role of Antioxidants in the Chemistry of Oxidative Stress: A Review." European Journal of Medicinal Chemistry 97:55-74. doi: 10.1016/j.ejmech.2015.04.040. 
Pluta-Kubica, Agnieszka, Ewelina Jamróz, Agnieszka Kawecka, Lesław Juszczak, \& Paweł Krzyściak. 2020. “Active Edible Furcellaran/Whey Protein Films with Yerba Mate and White Tea Extracts: Preparation, Characterization and Its Application to Fresh Soft Rennet-Curd Cheese." International Journal of Biological Macromolecules 155:1307-16. doi: 10.1016/j.ijbiomac.2019.11.102.

Poprac, Patrik, Klaudia Jomova, Miriama Simunkova, Vojtech Kollar, Christopher J. Rhodes, \& Marian Valko. 2017. “Targeting Free Radicals in Oxidative Stress-Related Human Diseases." Trends in Pharmacological Sciences 38(7):592-607. doi: 10.1016/j.tips.2017.04.005.

Ribas, Jessyca C. R., Paula T. Matumoto-Pintro, Ana Carolina P. Vital, Bianka R. Saraiva, Fernando A. Anjo, Ruth L. B. Alves, Nadine W. Santos, Erica Machado, Bruna C. Agustinho, \& Lúcia M. Zeoula. 2019. "Influence of Basil (Ocimum Basilicum Lamiaceae) Addition on Functional, Technological and Sensorial Characteristics of Fresh Cheeses Made with Organic Buffalo Milk.” Journal of Food Science and Technology 56(12):5214-24. doi: 10.1007/s13197019-03990-5.

Rochette, L., Steliana, G., Adriana, M., \& Catherine, V. 2015. "Alpha-Lipoic Acid: Molecular Mechanisms and Therapeutic Potential in Diabetes.” Canadian Journal of Physiology and Pharmacology 93(12):1021-27. doi: 10.1139/cjpp-2014-0353.

Saraiva, B. R., Vital, A. C. P., Anjo, F. A., Ribas, J. C. R., Paula, T. \& Matumoto-Pintro. 2019. Effect of yerba mate (Ilex paraguariensis A. St.-Hil.) addition on the functional and technological characteristics of fresh cheese. J Food Sci Technol 56, 1256-1265. doi:10.1007/s13197-019-03589-w

Sharma, G. N., Gaurav, G., \& Piyush, S. 2018. "A Comprehensive Review of Free Radicals, Antioxidants, and Their Relationship with Human Ailments." Critical Reviews ${ }^{T M}$ in Eukaryotic Gene Expression 28(2).

Shidfar, F., Shima, S. E., Sharieh, H., I. H., Shahrzad, S., \& Giti, H. 2012. "The Effects of Berberis Vulgaris Fruit Extract on Serum Lipoproteins, ApoB, ApoA-I, Homocysteine, Glycemic Control and Total Antioxidant Capacity in Type 2 Diabetic Patients." Iranian Journal of Pharmaceutical Research: IJPR 11(2):643-52.

Surai, P. F. 2020. “Antioxidants in Poultry Nutrition and Reproduction: An Update.”

Szymanska, R., Pavel, P., \& Jerzy, K. 2018. "Plant-Derived Antioxidants in Disease Prevention 2018." Oxidative Medicine and Cellular Longevity 2018:1-2. doi: $10.1155 / 2018 / 2068370$.

Thakur, P., Ashwini, K., \& Awanish, K. 2018. "Targeting Oxidative Stress through Antioxidants in Diabetes Mellitus.” Journal of Drug Targeting 26(9):76676. doi: 10.1080/1061186X.2017.1419478.

Tian, F., Eric, A. D., \& Julie M. G. 2013. "Controlling Lipid Oxidation of Food by Active Packaging Technologies.” Food \& Function 4(5):669. doi: $10.1039 / \mathrm{c} 3$ fo30360h.

Tureck, C., Gelvani L., Vanesa, G. C., \& Eloá, A. K. 2017. “Avaliação Da Ingestão de Nutrientes Antioxidantes Pela População Brasileira e Sua Relação Com o Estado Nutricional.” Revista Brasileira de Epidemiologia 20:30-42.

Vandamme, E. J., \& J. L. Revuelta. 2016. Industrial Biotechnology of Vitamins, Biopigments, and Antioxidants. Wiley.

Varadharaj, S., Owen, J. Kelly, R. N. Khayat, P. S. Kumar, N. A., \& Jay L. Z. 2017. "Role of Dietary Antioxidants in the Preservation of Vascular Function and the Modulation of Health and Disease." Frontiers in Cardiovascular Medicine 4:64. doi: 10.3389/fcvm.2017.00064.

Vassalle, C., Lorien S., A. Pingitore, K. Chatzianagnostou, F. Mastorci, R. Ceravolo. 2017. “Antioxidants in the Diet and Cognitive Function: Which Role for the Mediterranean Life-Style?” The Journal of Prevention of Alzheimer's Disease 4(1):58-64.

Zehiroglu, C., \& Sevim, B. O. S. 2019. “The Importance of Antioxidants and Place in Today’s Scientific and Technological Studies.” Journal of Food Science and Technology 56(11):4757-74. doi: 10.1007/s13197-019-03952-x.

Zemestani, M., Maryam R., \& Mohammad A. 2016. "Chamomile Tea Improves Glycemic Indices and Antioxidants Status in Patients with Type 2 Diabetes Mellitus.” Nutrition 32(1):66-72. doi: 10.1016/j.nut.2015.07.011.

Zhao, X., Ming Z., Chunxiao L., Xue J., Yana S., \& Ying Z. 2019. “Benefits of Vitamins in the Treatment of Parkinson's Disease.” Oxidative Medicine and Cellular Longevity 2019:1-14. doi: 10.1155/2019/9426867. 